\title{
Pleuro-Pulmonary Amoebiasis: About two New Case Reports
}

\author{
Zaghba $\mathrm{N}^{2}$, Bakkal $\mathrm{H}^{1 *}$, Benjelloun $\mathrm{H}^{2}$, Yassine $\mathrm{N}^{3}$
}

${ }^{1}$ Résidente En Pneumologie, Service des Maladies Respiratoires Du CHU Ibn Rochd, 1 Rue des Hôpitaux Casablanca 20360, Maroc

${ }^{2}$ Professeur De pneumologie, Service des Maladies Respiratoires Du CHU Ibn Rochd, 1 Rue des Hôpitaux Casablanca 20360, Maroc

${ }^{3}$ Professeur de pneumologie et chef de service des maladies respiratoires du CHU Ibn Rochd, 1 Rue des Hôpitaux Casablanca 20360, Maroc

DOI: $\underline{10.36347 / \text { sjmcr.2020.v08i07.024 }}$

| Received: 01.07.2020 | Accepted: 12.07.2020 | Published: 29.07 .2020

*Corresponding author: Hind Bakkal

Abstract

Case Report

Pleuro-pulmonary affection of amoebiasis is the most common extraintestinal site of amoebiasis after hepatic disorder. We report two case reports of pleuro-pulmonary amoebiasis concerning two men aged 47 and 37 years, respectively, with a history of dysentery syndrome. It is a purulent pleurisy in the first case associated with a hepatic abscess, and a parasitic eosinophilic lung in the second case. The diagnosis was confirmed by the high positive amoebic serology in the first case and on the radio-clinical data and the isolation of Entamoeba histolytica histolytica in the second with a good evolution under treatment. This was based on metronidazole $(1.5 \mathrm{~g} / \mathrm{d})$ for 14 days, with evacuation of the pleural cavity. The clinical and radiological progress was good in both cases.

Keywords: Amoebiasis; Entamoeba histolytica; Lung; Pleura; Diagnosis.

Copyright @ 2020: This is an open-access article distributed under the terms of the Creative Commons Attribution license which permits unrestricted use, distribution, and reproduction in any medium for non-commercial use (NonCommercial, or CC-BY-NC) provided the original author and source are credited.

\section{INTRODUCTION}

Amoebic pleuro-pulmonary disorder is the most common extraintestinal expression of amebiasis after liver disorder. The pathogen is a parasite: Entamoeba histolytica, the only pathogenic species for humans. We report 2 cases of pleuro-pulmonary amoebiasis, the diagnosis is underlined in light of clinical and radiological data and amoebic serology positivity and the detection of Entamoeba histolytica on stool parasitological examination. This work's interest is to insist on the clinical polymorphism of pleuropulmonary amoebiasis, on diagnosis means and therapeutic management.

\section{CASE RePORT 1}

Mr B.B., 47 years old, coming from and living in Casablanca, buildings painter, smoker of 20 packyears. He had two weeks earlier, right basi-thoracic pains with a dry cough and dyspnea worsening in progress in a context of health weakening, made of asthenia and weight loss not quantified with feverish sensations. He reported to be on travel recently but had noted a dysentery syndrome 2 months earlier. The clinical examination noted a right basi-thoracic fluid effusion syndrome with a fever of $38.5^{\circ} \mathrm{C}$ and a sensitivity of the right hypochondrium without other associated signs. The chest radiograph had shown right basal pleural type opacity. The pleural puncture had brought back a thick, non-fetid chocolate brown liquid, which tested for common germs and BK on direct examination and culture was negative. The search for amoebae in pleural fluid was negative. Blood count formula found out a leukocytosis at $12300 \mathrm{e} / \mathrm{mm} 3$ with a blood eosinophilia at $730 \mathrm{e} / \mathrm{mm} 3$.

Amoebic serology was positive by indirect hemagglutination (HAI) at 1/800 (point line 1/128) confirming the pleurisy amoebic origin. Thoracic drainage was performed to relieve the pleural cavity totaling $3500 \mathrm{cc}$ of thick chocolate brown liquid with lung return to the wall at D6 of drainage. The chest CT scan was unremarkable. The bronchoscopy showed a substantially normal endoscopic appearance. Moreover, abdominal ultrasound underlined two hepatic abscesses; the largest one was $8 \mathrm{~cm}$ long. The patient was administered metronidazole $1.5 \mathrm{~g} / \mathrm{d}$ with a good clinical improvement under treatment and hepatic abscess persistence whose puncture was scheduled after consultation with the gastroenterologists, as well as a thoracoabdominal MRI looking for a diaphragmatic fistula but the patient refused further investigation.

\section{Case RePort 2}

Mr AH 37 years old, bricklayer, of rural origin (southern Morocco), who repported to have effort dyspnea for 9 months before his hospitalization, associated with a dry cough, a fever at $38.5^{\circ} \mathrm{c}$ and asthenia without weight loss or digestive disorders. 
Interrogation found out no history of wheezing, no recent medication, or no recent trip. The physical examination was normal. The chest radiograph showed out fixed bilateral and symmetrical peripheral infiltrates in the middle regions, producing the appearance of mantle edema (Fig1a). The thoracic scan had revealed a frosted glass interesting especially the posterior and peripheral regions of the upper lobes, the middle lobe, and the upper part of the lower lobes (Fig1b).

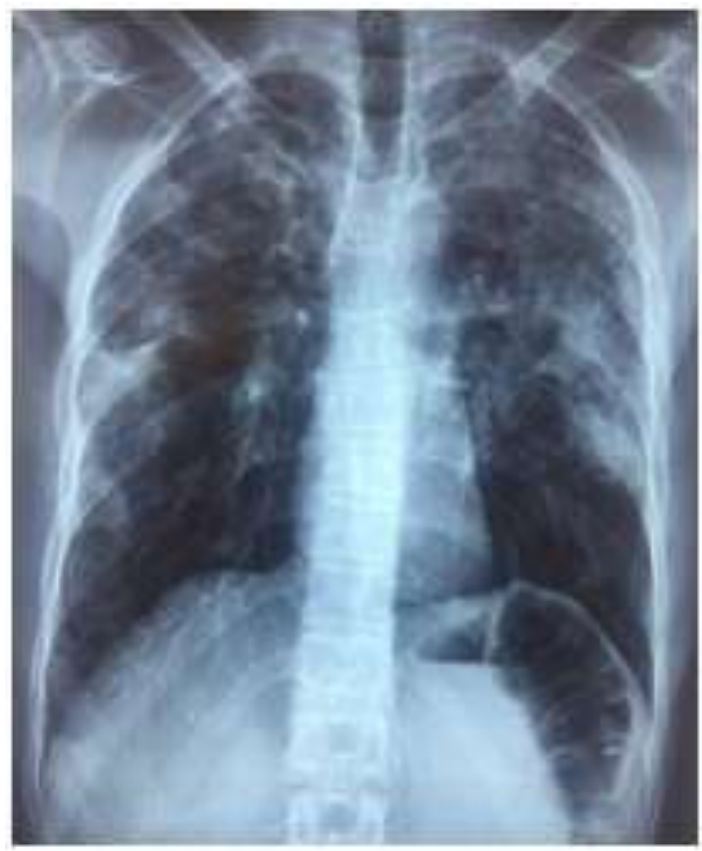

Fig-1a: Chest X-ray on admission

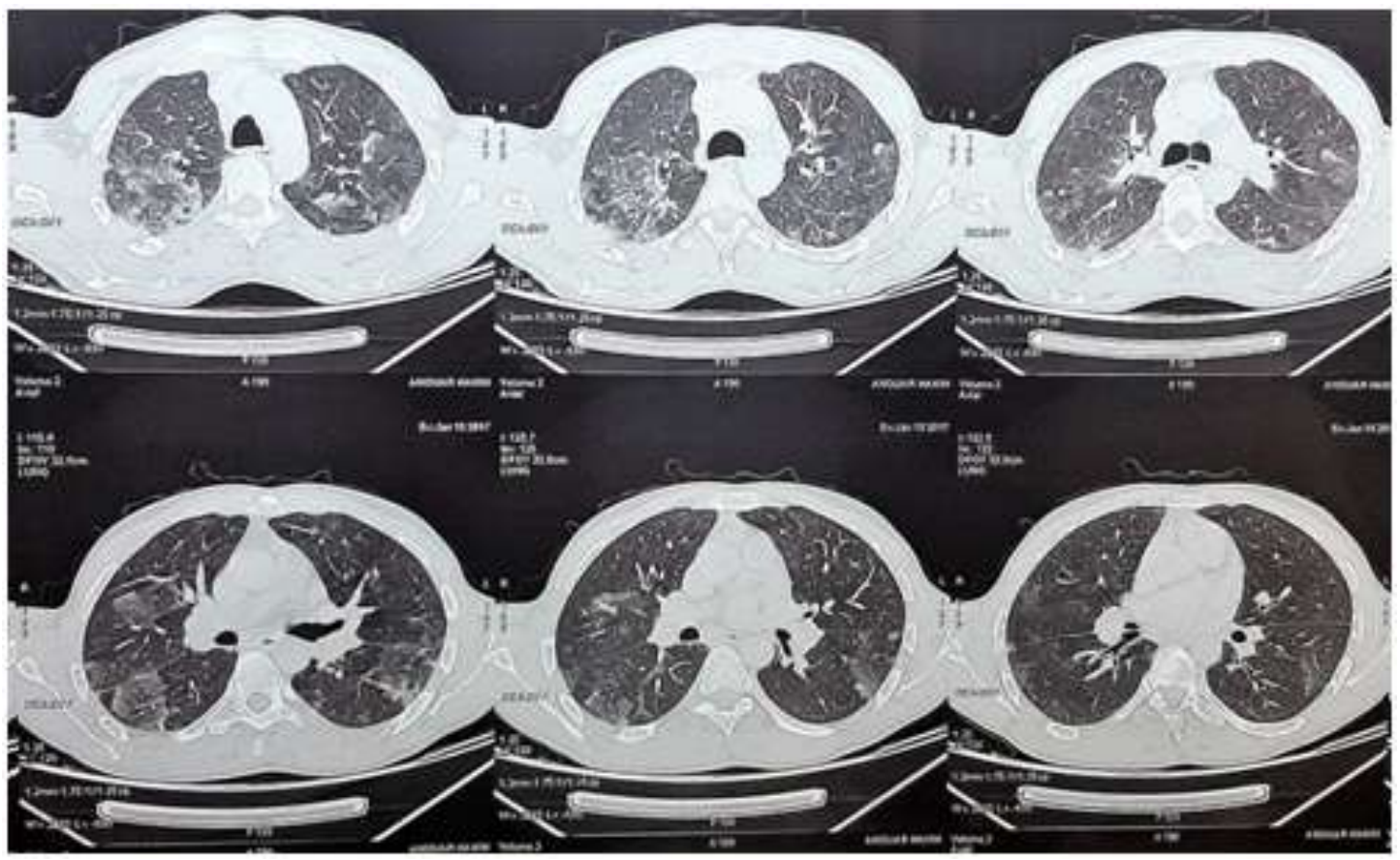

Fig-1b: Entrance chest scanner showing bilateral ground glass 

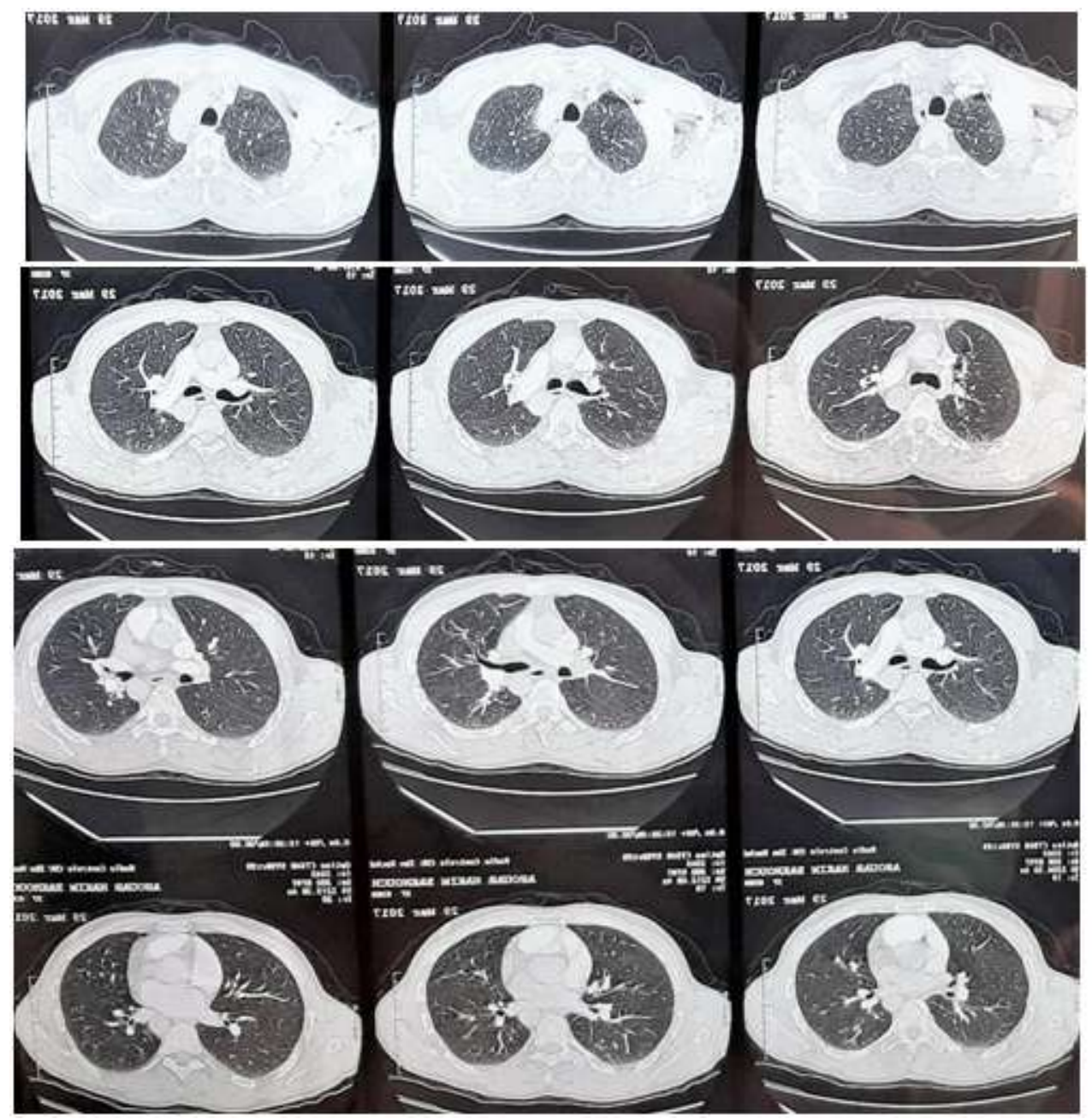

Fig-2: Chest CT after antiparasitic treatment

The blood count showed obvious hypereosinophilia at $2010 / \mathrm{mm} 3$ repeatedly controlled. On bronchoscopy, a first-degree inflammatory state was noted. The Genexpert in the bronchial aspiration fluid was negative and the bronchial biopsies were in favor of a moderate inflammatory infiltrate rich in eosinophilic polynuclear cells forming micro-abscesses in places. The bronchio-alveolar pump underlined a non-hematous macrophagic fluid with an eosinophilia at $9 \%$. At this stage, eosinophilic lung diagnosis was accepted.

For the etiological assessment of the eosinophilic lung, the ANCAs determination in search of vasculitis of the allergic granulomatous angiitis type was negative two times. The ENT examination, the blonde scanner and the renal check-up were normal. The results for sarcoidosis as well as for allergic bronchopulmonary aspergillosis were negative. Amoebic, ascaribiosis, bilharziasis and filariasis serology were negative. However, a parasitological stool examination (EPS) had isolated Entamoeba histolytica. It is therefore an eosinophilic parasitic lung of amoebic origin. No other visceral localization of amoebiasis had been found, mainly no abscess on the sub-diaphragmatic floor on abdominal ultrasound. The HIV serology was negative.

The patient was administered metronidazole at a rate of $1.5 \mathrm{~g} / \mathrm{D}$ for 14 days with reduction in hypereosinophilia without negation of the control EPS on D14 of the treatment, then he was taken up by albendazole $400 \mathrm{mg}$ dose unique, renewed 7 days later, with good outcome.

The outcome was very favorable with regression of clinical signs, negativity of EPS, normalization of eosinophilia and radiological cleaning with disappearance of the frosted glass at the end of the antiparasitic treatment (Fig 2).

\section{DISCUSSION}

Amoebiasis is a cosmopolitan parasitosis that predominates widely in the Third World hot countries of [1]. The responsible parasite is Entamoeba histolytica which is the only pathogenic species in humans among the six amoeba types identified up to date. Transmission is mainly oral-faecal or through contaminated food and water [2, 3]. Central America, Africa and South Asia are the most affected regions [3]. Patients with visceral 
amoebiasis have an average age of 40 years with a male predominance $[3,4]$.

It affects around 500 million people worldwide, $10 \%$ of whom have the intestinal invasive form and $1 \%$ who have the extra-intestinal invasive form, causing up to 100,000 deaths a year [3-5]. Indeed, it is the third cause of death from parasitosis in the world after malaria and schistosomiasis $[1,5,6]$; hence the importance of early diagnosis of its complications with intention of early treatment [4]. It is a reportable disease in some European countries [7].

E. histolytica Infection in its invasive form is more common in patients with human immunodeficiency virus, but the hepatic amoebiasis prevalence does not appear to be high in these patients, nor is it more severe [6].

This parasite's Primary infection results into acute amoebic colitis, which is mainly mild. Tissue infection is secondary to colonic amoebiasis, this is due to E. histolytica passage through the intestinal wall [6], which will be forwarded through the portal vein, to the liver leading to focal abacterial necrosis and then to abscess. If intestinal and colic infections are frequent, tissue infections are more rare but serious. Liver abscess is the most common extraintestinal complication followed by pleuro-pulmonary amoebiasis [4-6]. It can occur even in a subject living outside the endemic area [7], as is the case with our patients.

Hepatic localization represents 1 to $25 \%$ of invasive amebiasis [6] and pleuro-pulmonary disorder is often secondary to hepatic amoebiasis. It occurs in approximately $20 \%$ of patients with amoebic liver abscesses. This mechanism is explained by an intrathoracic passage, either by trans-phrenic propagation by diffusion or by intrusion of the hepatic abscess through the diaphragm (which explains the clear predominance of the right thoracic localizations), either by vascular propagation or by venous intrusion, or inhalation of $E$. cysts. Histolytica $[1,2,4,5,7,8]$. As a result, the amoebic lung abscess preferentially lies on the right but can also be on the left. Pleuro-pulmonary amoebiasis can be primary, related to a dissemination of the amoeba by blood with overflow of the hepatic filter; this is the case of several isolated visceral thoracic lesions without hepatic localization reported in the literature [1, 2, 4, 8]. Our second patient had an Entamoeba histolytica eosinophilic lung but had no associated liver damage.

Pleural disorder counts for 17 to $68 \%$ of the amoebiasis chest localizations [4]. It is often in the right seat, which is the case for our first patient.

The abdominal ultrasound should be systematic in case of purulent pleurisy or right basal pulmonary abscess to look for hepatic abscess, which would guide the diagnosis $[3,4,6]$.

Pleuro-pulmonary amoebiasis' radiological and clinical aspects are non-abscessed amoebic pneumonia, systematized (right lower lobe, right middle lobe) or non-systematized, pulmonary abscess complicated by pyothorax, amoebic pleurisy due to abscess rupture hepatic in the pleura, hepatobronchial fistulas, or less common forms such as pleural empyema or bilateral excavated nodular pneumonia [2, 5 , 9]. Our first patient had right purulent pleurisy associated with liver abscesses. The second one had an Entamoeba histolytica eosinophilic lung without liver damage, which is the particularity of our second case report.

Eosinophilic pneumonia is defined by respiratory symptoms associated with radiological infiltrates and the presence of peripheral eosinophilia greater than $1500 \mathrm{~g} / 1$ and / or alveolar $>25 \%$. They are divided into several entities according to clinical and etiological characteristics, the most common etiologies of which are parasites, including amoebiasis [10].

Visceral amoebiasis diagnosis is based on radio-clinical data. At the clinical stage, we look for the presence of an unhealthy lifestyle, a recent trip to an endemic area, a history of dysentery syndrome, the presence of associated febrile liver disease and the occurrence of 'a chocolate brown pus vomit. The chocolate brown appearance of the pleural fluid and the right basal thoracic seat suggest the diagnosis at the radio-clinical stage $[3,7]$. In addition, the pleural fluid can be citrine yellow, purulent or pyo-hematic $[1,4,8]$. Amoebic serology and identification of the pathogenic amoebic species confirms the diagnosis.

Amoebae are rarely detected in biological samples, pus or pleural fluid (less than $10 \%$ of cases) $(2,3,7)$. Amoebic serology is very sensitive (> 94\%) and specific (> 95\%) for visceral amoebiasis diagnosis by ELISA, HAI, latex test or indirect immunofluorescence (IFI) [2-4]. It is also a posttherapeutic monitoring means. False negative reactions may be found at the onset of infection (within the first 7-10 days) but will become positive later [4, 7]. Serology is positive in 85 to $95 \%$ of patients with invasive amebiasis, a value equal to or greater than $1 / 320$ is an argument in favor [7]. E. cysts. dispar presence does not cause the appearance of serum antibodies and asymptomatic carriage of E. histolytica is responsible for seroconversion in 25 to $45 \%$ of cases, whereas in the case of intestinal amebiasis, a significant level of circulating antibodies is detected in $70 \%$ of cases [3]. Among these techniques, the IFI has the best efficacy $(95.5 \%)$ compared to latex $(94.3 \%)$ and the ELISA $(93.5 \%)$ [3]. an alternative to serology is represented by antigenemia (detection of the lectin Gal/GalNAc specific for E. histolytica by ELISA 
technique and by RT-PCR [2]. In case of difficult interpretation of serologies, positive stool microscopic examination and coproantigens detection will be more appropriate. Only the detection of the hematophagous form of E. histolytica in stool by microscopy can confirm the diagnosis [3]. An inflammatory syndrome may be together with the symptomatology but the leukocytosis may be missing or discreet $[6,7]$, this is the case for our two patients.

Invasive amebiasis diagnosis was highlighted in our patient on the radio-clinical data and confirmed in the first case by amoebic positive serologies and in the last one by the isolation of Entamoeba histolytica histolytica in EPS.

Amoebiasis is a main cause of parasitic morbidity. Lethal ruptures of hepatic abscesses or pleural empyemas in the pericardium have already been reported mainly in patients with amoebic pleural empyemas on the left side, which makes this disease potentially fatal. Mortality can reach up to $15 \%$ in endemic areas [5, 6].

In addition, the evolution is generally good with mortality less than $1 \%$ if the diagnosis is early and after a well-managed treatment [1, 4-6].

The thoracic disorder of our patients has well progressed with antiparasitic treatment together with local treatment in the case of pleurisy.

The disease's primary prevention requires above all a healthy lifestyle based on drinking water availability and struggle against fecal peril.

\section{CONCLUSION}

Pleuro-pulmonary amoebiasis should be suspected in case of any pathology of infectious origin of the right thoracic base. It is often associated with a liver abscess. The diagnosis is based mainly on radioclinical elements and is confirmed by serology. It can be serious and requires early diagnosis and management for a good prognosis. In front of an eosinophilic lung of probable parasitic origin, we must think of Entamoeba histolytica which can be suggested even in the absence of a trip to the endemic area and in the absence of dysentery syndrome, as in the case of our patient. The evolution remains good in the case of well therapeutic management. Healthy living remains the best means of prevention against this disease.

\section{REFERENCES}

1. Rachid H, Yazidi AA, Loudadssi F, El Biaze M, Bakhatar A, Yassine N, Meziane AE, Bahlaoui A. Les atteintes pleuro-pulmonaires à Entamoeba histolytica. Revue des maladies respiratoires. 2005 Dec 1;22(6):1035-7.

2. http://medecinetropicale.free.fr/cours/parasitose_pu lmonaire.pdf.

3. Beyls N. Diagnostic sérologique de l'amibiase à Entamoeba histolytica: validation d'un nouveau test ELISA au CHU de Grenoble.

4. Makni F, Sellami H, sellami A, Cheikhrouhou F, Ayadi H, Benjemaa M. L'amibiase viscérale : bilan dans le CHU de sfax. Amoebiasis: study in sfax university hospital. Rev Tun Infectiol. 2007, 1(2): 20-24.

5. Pleural empyema secondary to amebic liver abscess. International Journal of Infectious Diseases 2009; 13: 135-136.

6. Soko TO, Ba PS, Carmoi T, Klotz F. Amibiase (amoebose) hépatique. EMC-Hépatologie. 2016: 19.

7. Hugard L, Ph. Dubrous, Massoure PL, Thefenne H and Coué JC. Amibiase pleuropulmonaire: un diagnostic inattendu. Bull Soc Pathol Exot. 2005, 98(2): 89-90.

8. Loudadsi F, El Biaze M, Bakhatar A, Rachid H, Yassine N, El Meziane A. Amibiase pleuropulmonaire. A propos de 3 cas. Rev Mal Respir. 2004; 21: 68.

9. Ayadi H, Rekik WK, Ayoub K. Pneumopathie nodulaire excavée bilatérale : l'amibe responsable ? Rev Pneumol Clin. 2001; 57(6): 434-7.

10. Djakovic, Tanja, Ribeiro, Diana, Brossard, Christophe. Pneumopathies à éosinophiles, comment les différencier: classification et démarche diagnostique. Rev médicale suisse. 2016; 539:1958-1965. 